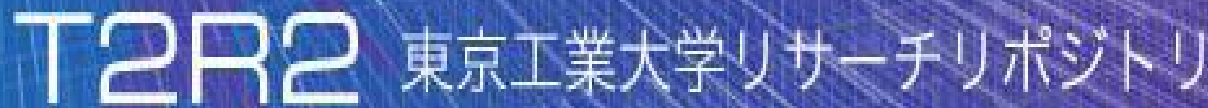

\section{Tokyo Tech Research Repository}

\section{論文 /著書情報 \\ Article /Book Information}

\begin{tabular}{|c|c|}
\hline Title & $\begin{array}{l}\text { Extreme ultraviolet light sources and soft x-ray laser based on } \\
\text { discharge produced plasma }\end{array}$ \\
\hline Authors & $\begin{array}{l}\text { E. Hotta, Y. Sakai, Y. Hayashi, G. Niimi, B. Huang, Q. Zhu, I. Song, M. } \\
\text { Watanabe }\end{array}$ \\
\hline Citation(English) & Proc. of SPIE, Vol. 9524, , pp. 9524U1- 8 \\
\hline 発行日 /Pub. date & 2015,8 \\
\hline DOI & http://dx.doi.org/10.1117/12.2195258 \\
\hline 権利情報 /Copyright & $\begin{array}{l}\text { 本著作物の著作権はSociety of P hoto-O ptical Instrumentation } \\
\text { Engineersに帰属します。 } \\
\text { Copyright } 2015 \text { Society of Photo-O ptical Instrumentation Engineers. } \\
\text { One print or electronic copy may be made for personal use only. } \\
\text { Systematic reproduction and distribution, duplication of any material in } \\
\text { this paper for a fee or for commercial purposes, or modification of the } \\
\text { content of the paper are prohibited. }\end{array}$ \\
\hline
\end{tabular}


Invited Paper

\title{
Extreme ultraviolet light sources and soft x-ray laser based on
}

\section{discharge produced plasma}

\author{
Eiki Hotta*, Yusuke Sakai, Yasushi Hayashi, Gohta Niimi, \\ Bin Huang, Qiushi Zhu, Inho Song, and Masato Watanabe \\ Department of Energy Sciences, Interdisciplinary Graduate School of Science and Engineering, \\ Tokyo Institute of Technology \\ Nagatsuta 4259 J2-35, Midori-ku, Yokohama 226-8502, Japan
}

\begin{abstract}
Due to the demand to realize shorter wavelength light sources, extreme ultraviolet (EUV) sources and soft x-ray laser (SXRL) are under development. The development of EUV sources at the wavelength of 13.5 nm started to realize light sources to be used for next generation lithography. Xenon was used at the beginning of development, however, to attain higher conversion efficiency, tin is now used as fuel. As a coherent light source, capillary discharge SXRL is under development. After the demonstration of Ne-like Ar SXRL by using electron collisional excitation scheme, the effort to shorten the wavelength has been made by adopting recombination scheme such as H-like N. Though the challenge has not yet been successful, the source has potential to be used as a SXR source in the water window wavelength region. Current status of EUV and SXR sources based on discharge produced plasma will be given.
\end{abstract}

EUV source, SXR laser, Capillary discharge, Z-pinch, Discharge produced plasma

\section{INTRODUCTION}

To meet the requirement for the next generation lithography, the research and development of 13.5 nm extreme ultraviolet (EUV) light sources based on discharge produced plasma (DPP) has been energetically pushed forward for the last decade. Although the research started from a xenon (Xe) capillary DPP EUV source, to reduce the interaction between plasma and the capillary inner wall, a Xe gas jet EUV source without a capillary was developed and the formation of EUV emission plasma with a small diameter at discharge current of $10 \mathrm{kA}$ was successfully realized. The obtained EUV conversion efficiency of Xe plasma is less than 1\%. In order to increase the conversion efficiency, which leads to the reduction of thermal load on the discharge head, a laser assisted DPP tin (Sn) EUV source has been also studied. It was demonstrated

\footnotetext{
*Corresponding author: ehotta@es.titech.ac.jp
}

International Conference on Optical and Photonic Engineering (icOPEN 2015), edited by Anand K. Asundi, Yu Fu, Proc. of SPIE Vol. 9524, 95242U · C 2015 SPIE · CCC code: 0277-786X/15/\$18 · doi: 10.1117/12.2195258 
that the position as well as the pinch time of EUV emission plasma could be controlled by laser irradiation energy according to the electrode gap length. Due to the problem of EUV output power scalability to a commercial source, DPP EUV sources are currently limited to be used as sources for trial manufactures or diagnoses of the illumination optics. However, to realize higher power DPP light sources with shorter wavelength, understanding of the Z-pinch plasma dynamics is essential.

Laser is a well-known light source which has narrow band width, high coherence and directivity. The wavelength of laser has also been shortened to soft $x$-ray region after vigorous research for a few decades ${ }^{[1]}$. The first capillary discharge soft x-ray laser (SXRL) has been demonstrated by J. J. Rocca's group in 1994 by using Ne-like Ar at the wavelength of $46.9 \mathrm{~nm}{ }^{[2]}$. Among several groups who succeeded the Rocca's group, our group revealed that the stable operation of SXRL could be realized in axially uniform plasma column obtained by applying appropriate pre-discharge for suppression of the growth of magnetohydrodynamic (MHD) instabilities in implosion phase ${ }^{[3]}$. To shorten the wavelength of SXRL, H-like $\mathrm{N}$ recombination laser at $13.4 \mathrm{~nm}$ has been proposed ${ }^{[4]}$ and is under investigation.

Capillary discharge plasma has steep electron density gradient at and after maximum pinch, which leads to the divergence of x-ray. Therefore, to realize a strong amplification in high density capillary discharge plasma, wave guide effect obtained by controlling the electron density distribution must be used ${ }^{[5]}$. The wave guide effect is especially important for the recombination scheme because the lasing may occur in expansion phase after the maximum pinch in which convex electron density distribution is usually generated and maintained.

\section{EXTREME ULTRAVIOLET SOURCES}

\subsection{Xenon Gas Jet DPP EUV Source}

A schematic of experimental setup, in which a double nozzle gas jet type electrode is adopted, is shown in Figure 1. Xenon (Xe) gas pre-ionized by inductively coupled RF power is supplied through an inner nozzle to form a fuel gas jet in between a cathode and an anode, and the outer nozzle is used for a coaxial He gas curtain to mitigate debris. EUV emitted from the pinched Xe DPP is supposed to be collected in radial direction. The unique feature of the electrode structure is that a capillary, which is usually used and becomes a source of debris, is removed. Even if debris is generated, the curtain gas takes away it downstream. A pulsed power supply with magnetic pulse compression provides a single current pulse of about $10 \mathrm{kA}$, which is shown in Figure 2.

The combination of a Si/Zr coated photodiode (IRD AXUV20HS1) and a $50 \mu \mathrm{m}$ pinhole is mounted to record the time resolved EUV radiation from 11 to $18 \mathrm{~nm}$. Figure 2 shows the measured EUV output signals with and without a He gas curtain. EUV emission increases with a He gas curtain, the cause of which may be attributed to the confinement of Xe gas by the gas curtain and the resulted change of pinch dynamics. The adoption of a diffuser increases EUV emission further. The ambient gas pressure in the vacuum chamber decreases when the diffuser operates, which may lead to less absorption of EUV by the ambient gas. The measured energy conversion efficiency (CE) from the input electric energy to EUV 


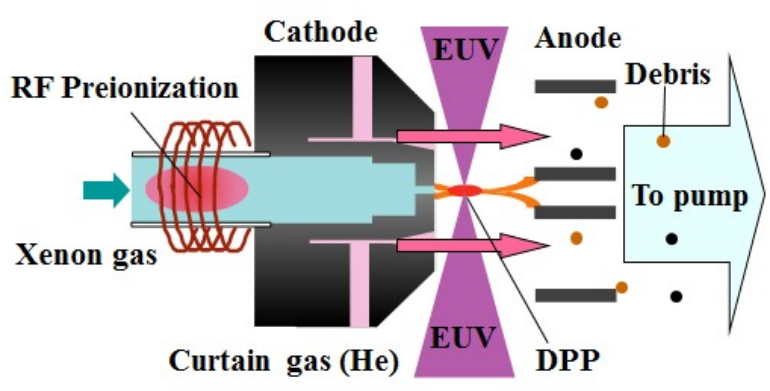

Figure 1 Schematic of gas-jet Z-pinch light source

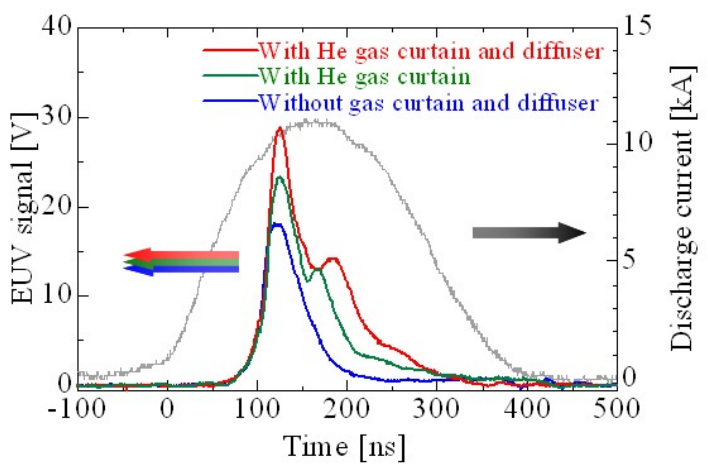

Figure 2 Wave forms of discharge current and EUV output signals with and without gas curtain

emission energy was less than $1 \%$.

\subsection{Laser-assisted Tin DPP EUV Source}

To increase the energy conversion efficiency, tin (Sn) is currently used as fuel material instead of Xe because Sn has higher CE. As Sn is solid at room temperature, it must be evaporated before discharge. By use of a Q-switched Nd:YAG laser the surface of a Sn rod buried in a cathode holder was irradiated and the ablated Sn plume was used as fuel. This type of discharge plasma is called laser-assisted discharge produced plasm (LADPP). The schematic of the system is shown in Figure 3.

The electrodes are set in a vacuum chamber and a high voltage of $-20 \mathrm{kV}$ is applied to the cathode. The cathode is directly connected to a ceramic capacitor bank of $80 \mathrm{nF}$. When a laser having output energy less than $135 \mathrm{~mJ}$ is irradiated to the Sn surface through a focusing lens, the generated Sn plasma plume expands toward the anode. The gap distance between electrodes is $4 \mathrm{~mm}$. By bridging the electrodes by the plasma plume a current up to $17 \mathrm{kA}$ with a rise time of $110 \mathrm{~ns}$ begins to flow through the plume and Sn plasma implodes by Z-pinch. The electrode system acts just like a laser triggered vacuum switch, therefore no switch is connected in series in the electric circuit. This contributes to reduce the inductance of the discharge circuit.

To observe the plasma behavior during the discharge and to get 2D electron density distribution, time resolved shadowgraphy and Normarski interferometry were employed. EUV emission image was recorded with a time integrated EUV pinhole camera which consisted of a Zr filter and a CCD. The in-band (2\%BW@13.5 nm) EUV output was measured with a calorimeter, which consisted of a Zr filter, a Mo/Si mirror and an X-ray photodiode (AXUV20HS1, IRD). The details of experimental setup and the diagnostic systems can be found elsewhere ${ }^{[6]}$.

Figure 4 shows the current and EUV emission waveforms. Electric input energy into plasma estimated by the measured 
current and voltage was $544 \mathrm{~mJ} / \mathrm{shot}$ and the measured in-band EUV energy was $8.9 \mathrm{~mJ} /(2 \pi \mathrm{sr}) / \mathrm{pulse}$, therefore CE is 1.65\%. Figure5 shows the time integrated in-band EUV emission images obtained by different shots. The strong EUV emission region is spatially stable shot by shot. The diameter of the higher EUV emission region is less than $1 \mathrm{~mm}$, which is small enough as a light source, that is, LADPP EUV source has small etendue. Figure 6 shows the electron density distribution at maximum pinch (at $190 \mathrm{ns)}$ measured with Normarski interferometer. The electron density related to the maximum EUV emission is $(2-3) \times 10^{18} \mathrm{~cm}^{-3}$ which is within the optimum density for the in-band EUV emission given by simulation result ${ }^{[7]}$.

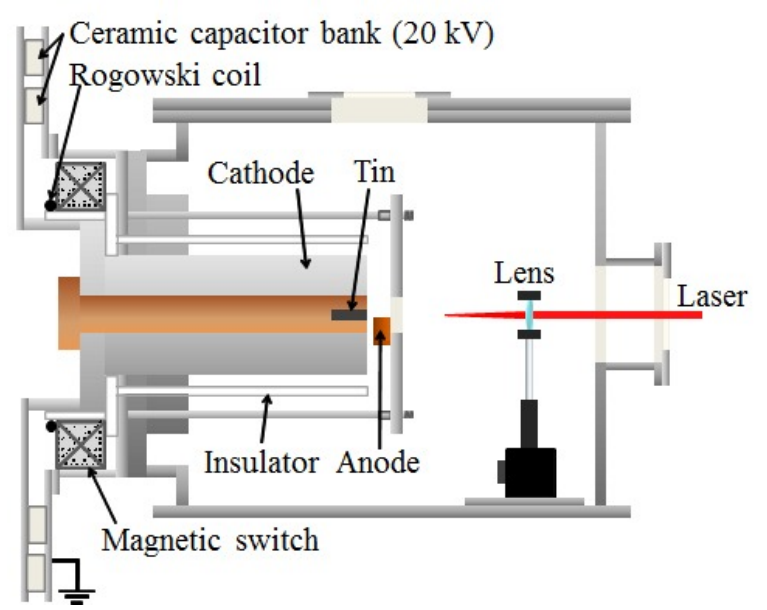

Figure 3 Schematic of LADPP EUV source

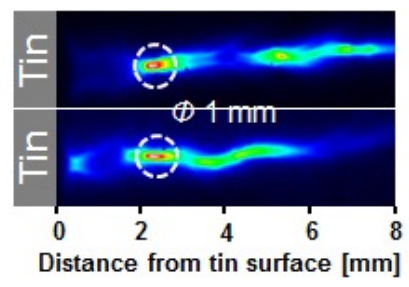

Figure 5 Time integrated in-band EUV image

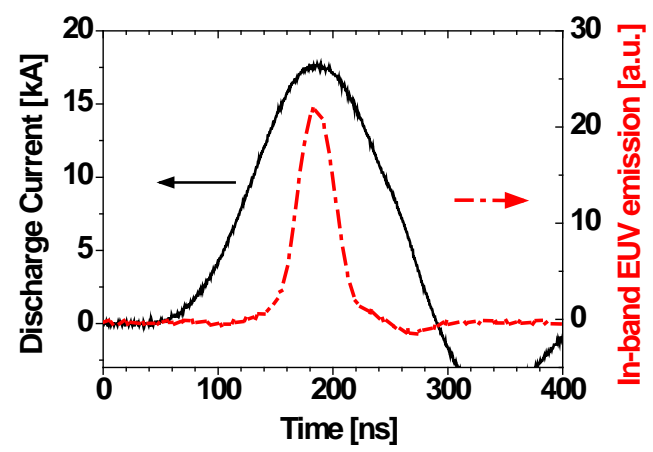

Figure 4 Current and EUV emission waveforms

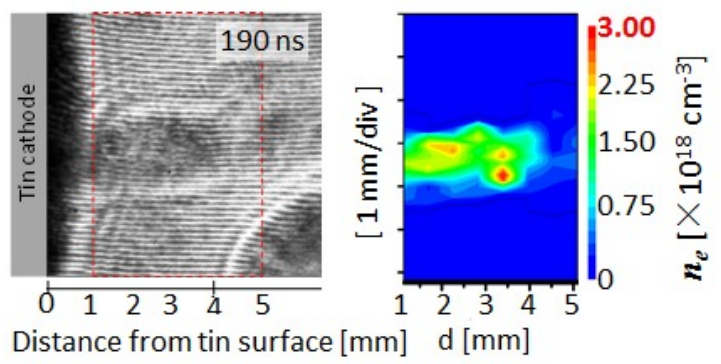

Figure 6 Electron density distribution at $190 \mathrm{~ns}$

\section{SOFT X-RAY LASER}

\subsection{Ne-like Ar soft X-ray laser}

Ne-like Ar soft X-ray laser (SXRL) at the wave length of $46.9 \mathrm{~nm}$ based on collisional excitation has been developed by using a ceramic capillary and a pulsed power generator. The details of the experimental setup can be found elsewhere ${ }^{[3]}$. Lasing has been successfully observed in wide range of Ar pressure from 150 to 750 mTorr according to the amplitude of discharge current up to $32 \mathrm{kA}$. The minimum current at which lasing was confirmed is $9 \mathrm{kA}$. The importance of preionization to get lasing, which prevents the growth of magnetohydrodynamic (MHD) instabilities by generating axially 
uniform plasma column, was also revealed ${ }^{[3]}$. Figure 7 shows the dependence of SXRL output on capillary length. Laser output saturation occurs beyond the capillary length of $180 \mathrm{~mm}$. The maximum gain-length product obtained in this case is 5. Figure 8 shows the SXRL output measured with an end-on x-ray diode (XRD) by using two capillaries with inner diameter of 3 and $4 \mathrm{~mm}$ and length of $180 \mathrm{~mm}$ at discharge current of $23.5 \mathrm{kA}$. Higher SXRL output was anticipated by obtainable larger amount of excited ions for $4 \mathrm{~mm}$ capillary, however the obtained SXRL output is lower than that obtained by using a $3 \mathrm{~mm}$ capillary. Even though the reason is not known, the pre-ionization and the resultant pinch dynamics may affect the result.

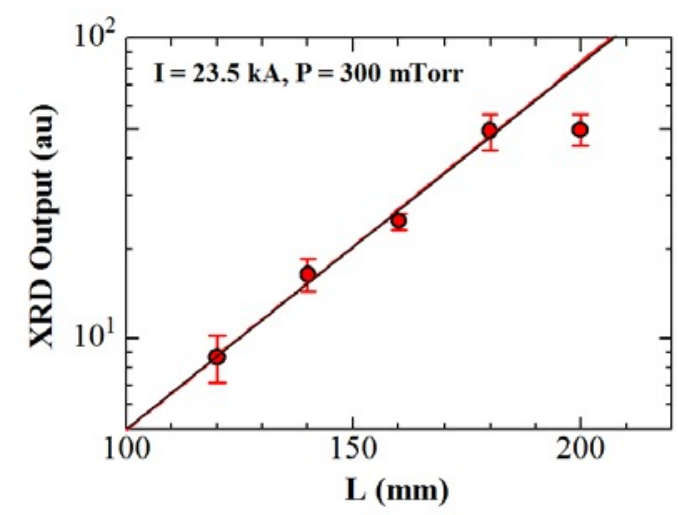

Figure 7 Dependence of SXRL output on capillary length

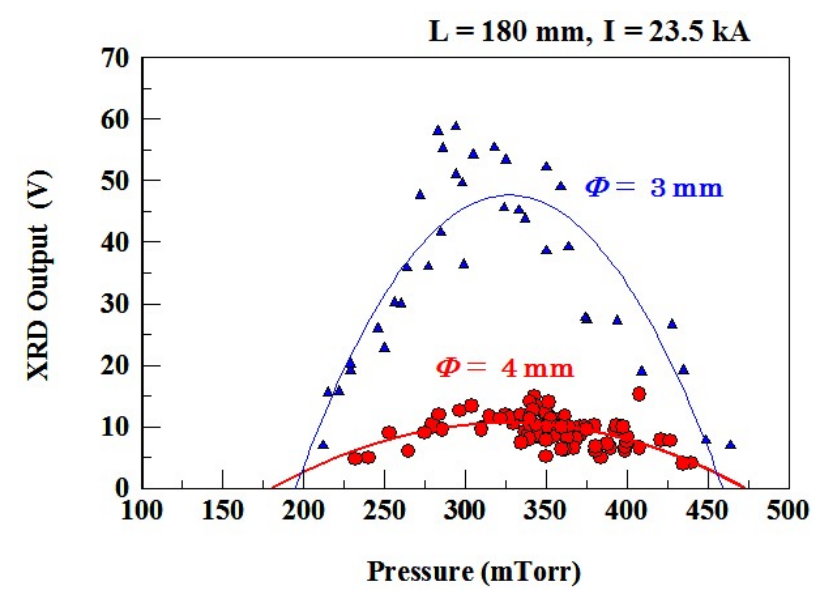

Figure 8 XRD output obtained by capillaries of 3 and $4 \mathrm{~mm}$ in diameter

\subsection{Capillary discharge nitrogen plasma}

Toward the realization of a much shorter wave length SXRL by using recombination scheme, $\mathrm{H}$-like $\mathrm{N}$ recombination laser at $13.4 \mathrm{~nm}$ (Balmer $\alpha$ ) has been proposed ${ }^{[4]}$. In general required input power for SXRL is inversely proportional to fourth power of wavelength. This means that to generate H-like N plasma, much higher electron temperature than that of Ne-like Ar SXRL is required, which leads to much higher discharge current. Therefore, a pulsed power supply capable of higher current up to $70 \mathrm{kA}$ with shorter pulse width of 60 ns was developed by using superposition of reflected voltage waves in a transmission line ${ }^{[8]}$. The electric circuit and schematic of the capillary discharge system is shown in Figure 9.

Nitrogen plasma was generated by a pulsed current having a peak amplitude of $50 \mathrm{kA}$ and a pulse width of $60 \mathrm{~ns}$. An alumina ceramic capillary with an inner diameter of $3 \mathrm{~mm}$ and a length of $75 \mathrm{~mm}$ was filled with nitrogen molecule gas. The spectrum of nitrogen plasma was recorded by a time-integrated spectrum pin-hole imaging set-up which consisted of a transmission grating with a lattice constant of $1 / 1000 \mathrm{~mm}^{-1}$ in combination with an X-ray CCD camera (DO434, Andor). Two slits $(1 \mathrm{~mm}$ x $5 \mathrm{~mm})$ were placed between the capillary and the transmission grating. The aperture diameter of the 


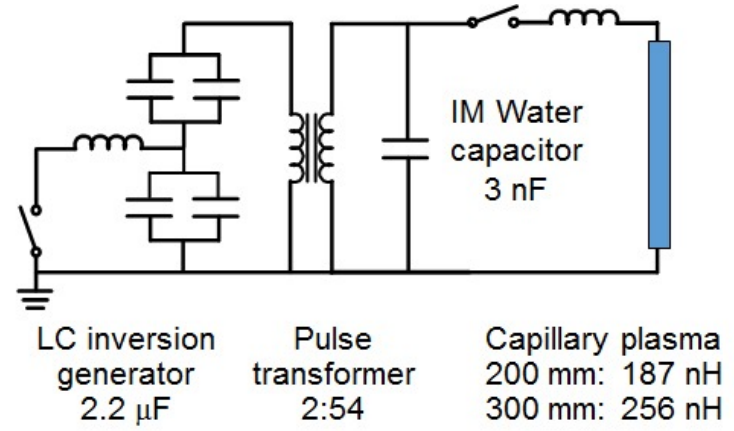

(a)

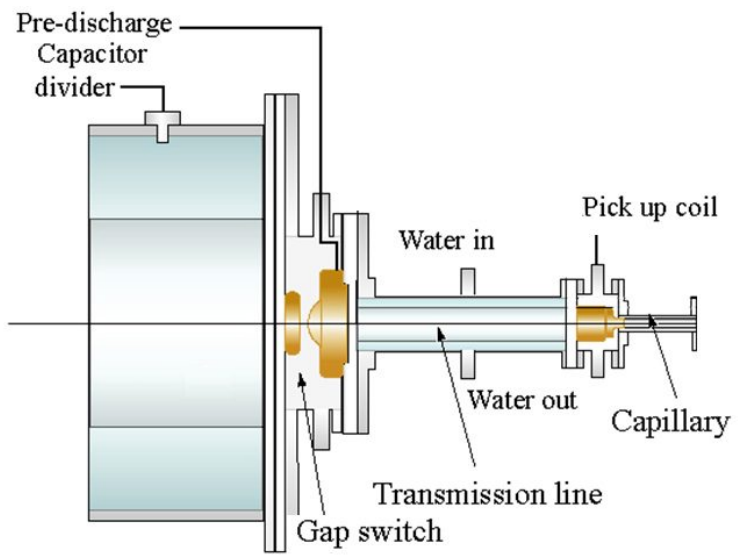

(b)

Figure 9 (a) Electric circuit and (b) schematic of capillary discharge system with a water transmission line

transmission grating was $50 \mu \mathrm{m}$. The resulted spectral resolution for a wavelength of $10 \mathrm{~nm}$, SXR is $\sim 0.2 \mathrm{~nm}$ and the radial special resolution is $\sim 10 \mu \mathrm{m}{ }^{[9]}$. Figure 10 shows the on-axis spectrum at various initial pressure and current amplitude of $50 \mathrm{kA}$, which are expected to have information at maximum pinch. The local electron temperature is estimated by fitting the slope of the bremsstrahlung radiation ${ }^{[10]}$. Maximum electron temperature of about $150 \mathrm{eV}$ was obtained at $750 \mathrm{mTorr}$, however no distinct line spectrum at $13.4 \mathrm{~nm}$ could be observed. The causes are considered that the electron temperature is not enough to get a large amount of fully stripped nitrogen ions at the maximum pinch and the rays are refracted when they are passing through a plasma column with convex electron density distribution after maximum pinch. To control and make concave electron density distribution, application of axial magnetic field was tested expecting that the magnetic pressure of compressed axial magnetic field expels electrons on axis. Figure 11 shows the XRD signal recorded when axial

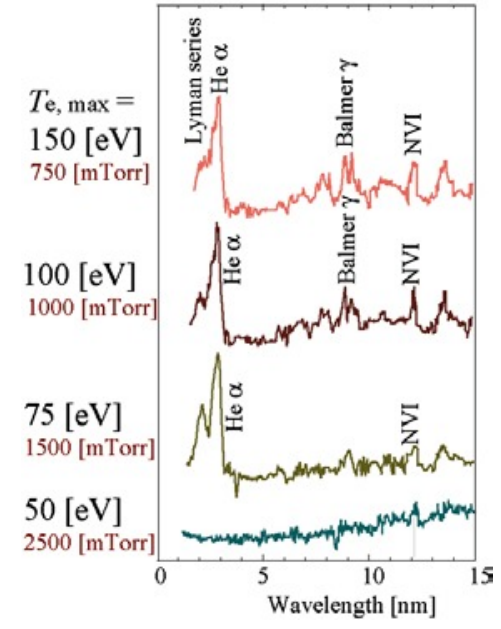

Figure 10 On-axis spectrum at various initial pressure, which are expected to have information at maximum pinch

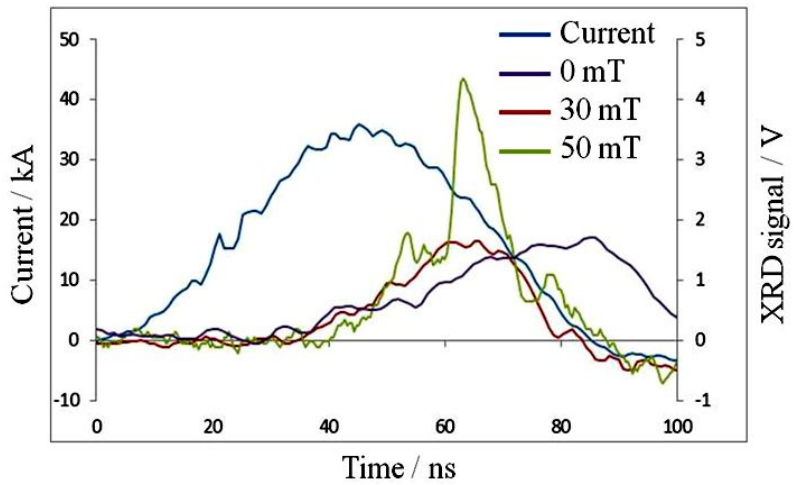

Figure 11 Current and XRD signal waveforms ( $p=200$ mTorr ) observed when axial magnetic fields are applied 
magnetic field is applied. For the initial magnetic flux density of $50 \mathrm{mT}$, increased signal can be observed. Since no further detailed experiments could be conducted, the reason is not clear, however convex electron distribution may be generated and this affected the transmission of SXR by wave guide effect. From Figure 10 strong emission at $2.49 \mathrm{~nm}$ of He-like N can be observed. This indicates that capillary nitrogen plasma can be used as a SXR source in the wavelength region of water window $(2.3-4.4 \mathrm{~nm})^{[11]}$ which is very useful for living cells observation.

\section{CONCLUSION}

EUV sources are under development to realize a debris free high power sources with higher conversion efficiency (CE) for next generation lithography. A xenon gas jet type DPP EUV source without a capillary demonstrated successfully the formation of EUV emitting high density high temperature z-pinch plasma, though CE is low. A laser-assisted tin DPP source has also shown the formation of pinched plasma, which has higher CE than that of Xe DPP source. In our case the attained CE is around $1.6 \%$. However, due to problems concerning scalability to the commercial EUV source of which output power is more than $200 \mathrm{~W}$, recently laser produced plasma (LPP) EUV sources are intensively developed and are adopted for a beta machine such as NXE3300B, ASML. To attain the required EUV power of more than $200 \mathrm{~W}$ at the intermediate focus, further research is required. As a coherent light source, capillary discharge soft X-ray lasers are also under investigation. The first capillary discharge SXRL was realized by Ne-like Ar at $46.9 \mathrm{~nm}$. The attempt to shorten the

laser wavelength is conducted by using $\mathrm{H}$-like $\mathrm{N}$ recombination scheme at $13.4 \mathrm{~nm}$. The attempt has not yet been successful, however the potential as a SXR source in the water window region $(2.3-4.4 \mathrm{~nm})$ was confirmed.

\section{Acknowledgment}

This work was supported by NEDO, EUVA and JSPS KAKENHI Grant Number 26630108.

\section{References}

[1] Elton, R. C., [X-ray Lasers], Academic Press, Boston (1990)

[2] Rocca, J. J., Shlyaptsev, V., Cortazar, O. D., Hartshorn, D., and Chilla, J. L. A. , "Demonstaration of a Discharge Pumped Table-Top Soft X-Ray Laser”, Phys. Rev. Lett. 73(6), 2129-2195 (1994)

[3] Niimi, G., Hayashi, Y., Sakamoto, N., Nakajima, M., Okino, A., Watanabe, M., Horioka, K. and Hotta, E., “Development and Characterization of a Low Current Capillary Discharge for X-ray Laser Studies”, IEEE Trans. Plasma Sci. 30(2), 616-621 (2002)

[4] Vrba, P., Vrbova, M., Bobrova, N. A., and Sasorov, P. V., "Modeling of a nitrogen x-ray laser pumped by capillary discharge”, Central European J. Phys. 3(4), 564-580 (2005)

[5] Suckewer, S., Skinner, C. H., Milchberg, H., Keane, C. and Voorhees, D., “Amplification of Stimulated Soft-X-Ray Emission in a Confined Plasma Column”, Phys. Rev. Lett. 50(17), 1753-1756 (1985)

[6] Zhu, Q., Yamada, J., Kishi, N., Watanabe, M., Okino, A., Horioka, K., and Hotta, E., "Investigation of the dynamics of the Z-pinch imploding plasma for a laser-assisted discharge-produced Sn plasma EUV source”, J. Phys. D: Appl. 
Phys. 44, 145203 (2011)

[7] Masnavi, M., Nakajima, M., Hotta, E., Horioka K., Niimi, G., and Sasaki, A., “Estimation of optimum density and temperature for maximum efficiency of tin ions in Z discharge extreme ultraviolet sources”, J. Appl. Phys. 101, 033306 (2007)

[8] Sakai, Y., Takahashi, S., Watanabe, M., Kim, G.-H., and Hotta, E., "Pulsed current wave shaping with a transmission line by utilizing superposition of a forward and a backward voltage wave for fast capillary Z-pinch discharge”, Rev. Sci. Instr. 81, 043504 (2010)

[9] Sakai, Y., Rosenzweig, J., Kumai, H., Nakanishi, Y., Ishizuka, Y., Takahashi, S., Komatsu, T., Xiao, Y., Bin, H., Zhu, Q., Hayashi, Y., Song, I., Kawamura, T., Watanabe, M., and Hotta, E., “Observation of emission process in hydrogen-like nitrogen Z-pinch discharge with time integrated soft X-ray spectrum pinhole image ”, Phys. Plasmas 20, 023108 (2013)

[10] Griem, H. R., [Plasma Spectroscopy], McGraw-Hill (1964)

[11] Vrba, P., Zakharov, S. V., Jancarek, A., Vrbova, M., Nevkla, M., and Kolar, P., "Pinching capillary discharge as a water window radiation source”, J. Elec. Spectroscopy and Related Phenomena, 184, pp.335-337 (2011) 